\title{
A epidemia HIV em adultos jovens na perspectiva da epidemiologia crítica
}

\author{
The HIV epidemics in young adults from the perspective of critical epidemiology \\ La epidemia de VIH en adultos jóvenes desde la perspectiva de la epidemiología crítica
}

Recebido: 19/12/2021 | Revisado: 27/12/2021 | Aceito: 01/01/2022 | Publicado: 02/01/2022

\author{
Marlise Lima Brandão \\ ORCID: https://orcid.org/0000-0002-2367-2390 \\ Universidade Federal do Paraná, Brasil \\ E-mail: marlise.brandao.ufpr@gmail.com \\ Maria Marta Nolasco Chaves \\ ORCID: https://orcid.org/0000-0001-8952-5695 \\ Universidade Federal do Paraná, Brasil \\ E-mail: mnolascochaves@gmail.com \\ Flaviane Marizete Limas \\ ORCID: https://orcid.org/0000-0002-2718-5143 \\ Universidade Federal do Paraná, Brasil \\ E-mail: flavianemlimas@gmail.com \\ Jorge Vinícius Cestari Felix \\ ORCID: https://orcid.org/0000-0002-0086-674X \\ Universidade Federal do Paraná, Brasil \\ E-mail: jvcfelix@ufpr.br \\ Daiane Siqueira de Luccas \\ ORCID: https://orcid.org/0000-0002-9173-8128 \\ Universidade Federal do Paraná, Brasil \\ E-mail: enfdailuccas@gmail.com \\ Rafaela Gessner Lourenço \\ ORCID: https://orcid.org/0000-0002-3855-0003 \\ Universidade Federal do Paraná, Brasil \\ E-mail: rglufpr@gmail.com \\ Jessyca Slompo Freitas \\ ORCID: https://orcid.org/0000-0002-0746-732X \\ Universidade Estadual do Centro-Oeste, Brasil \\ E-mail: jessyca_sfreitas@hotmail.com
}

\begin{abstract}
Resumo
Objetivo: Discutir a epidemia do HIV em adultos jovens, moradores dos municípios da Segunda Regional de Saúde do Paraná, notificados no período de 2007-2015. Metodologia: Estudo ecológico retrospectivo. Os dados foram coletados em sites públicos para caracterizar o território e o modo de viver da população local. As informações das notificações foram coletadas no SINAN-Net. A categoria de análise foi o modo de viver no território, ancorada nos pressupostos da Epidemiologia Crítica. Resultados: Foram identificadas 2.482 notificações em adultos de 20-29 anos, desses, 2.376 (95,7\%) eram casos de HIV e 106 (4,3\%), casos de aids. Ressalta-se que 74,3\% dos indivíduos declararam-se brancos e 95,9\% moravam em zona urbana. Quanto à ocupação do indivíduo, 59,1\% das notificações tiveram o campo não preenchido/ignorado. Considerações finais: $\mathrm{O}$ fenômeno HIV em jovens apresentou as maiores taxas de prevalência nos municípios com os piores indicadores sociais e os municípios que contavam com melhor infraestrutura dos serviços de saúde registraram o maior número de casos no período. Ações intersetoriais que reflitam sobre indicadores econômicos das sociedades e os índices epidemiológicos, comportamentos individuais e coletivos são necessárias para o enfrentamento do fenômeno.
\end{abstract}

Palavras-chave: HIV; Aids; Epidemiologia; Saúde coletiva; Enfermagem.

\begin{abstract}
Objective: To discuss the HIV epidemic in young adults, residents of the municipalities of the Second Health Regional of Paraná, notified in the period 2007-2015. Methodology: Ecological retrospective study. Data were collected from public websites to characterize the territory and the way of life of the local population. The information of the notifications was collected in SINAN-Net. The category of analysis was the way of living in the territory anchored in the assumptions of Critical Epidemiology. Results: We identified 2,482 notifications in adults aged 20-29 years, of these 2,376 (95.7\%) were cases of HIV and $106(4.3 \%)$ cases of AIDS. It is noteworthy that $74.3 \%$ of the individuals were white and $95.9 \%$ lived in urban areas. As for the individual's occupation, $59.1 \%$ of the notifications had the field not filled in/ignored. Final considerations: The HIV phenomenon in young people showed the highest prevalence rates in the municipalities with the worst social indicators and the municipalities that had the best
\end{abstract}


infrastructure of health services recorded the highest number of cases in the period. Intersectoral actions that reflect on the economic indicators of societies and the epidemiological indices, individual and collective behaviors are necessary to confront the phenomenon.

Keywords: HIV; Aids; Epidemiology; Public health; Nursing.

\section{Resumen}

Objetivo: Discutir la epidemia del VIH en adultos jóvenes, moradores de los municipios de la Segunda Regional de Salud de Paraná, notificados en el período 2007-2015. Metodología: Estudio ecológico retrospectivo. Se recogieron datos en sitios web públicos para caracterizar el territorio y el modo de vida de la población local. La información de las notificaciones se recogió en SINAN-Net. La categoría de análisis fue la forma de vivir en el territorio anclada en los supuestos de la Epidemiología Crítica. Resultados: Se identificaron 2.482 notificaciones en adultos de 20 a 29 años, de las cuales 2.376 (95,7\%) fueron casos de VIH y 106 (4,3\%) casos de SIDA. Cabe destacar que el 74,3\% de los individuos eran blancos y el 95,9\% vivían en zonas urbanas. En lo que respecta a la ocupación del individuo, el $59,1 \%$ de las notificaciones tenían el campo sin rellenar/ignorado. Consideraciones finales: El fenómeno del VIH en los jóvenes mostró las mayores tasas de prevalencia en los municipios con los peores indicadores sociales y los municipios que tenían la mejor infraestructura de servicios de salud registraron el mayor número de casos en el período. Las acciones intersectoriales que reflejan los indicadores económicos de las sociedades y los índices epidemiológicos, los comportamientos individuales y colectivos son necesarios para hacer frente al fenómeno.

Palabras clave: VIH; SIDA; Epidemiología; Salud pública; Enfermería.

\section{Introdução}

O Vírus da Imunodeficiência Humana (HIV) tem como alvo as células do sistema imunológico, e sua ação se divide em três fases: aguda (até quatro semanas após a infecção); assintomática ou latência (de oito a dez anos); Síndrome da Imunodeficiência Adquirida (aids), que surge com a perda da capacidade de resposta do sistema de defesa e aparecimento das doenças oportunistas (Brasil, 2017a).

A epidemia de HIV destaca-se entre as enfermidades infecciosas emergentes e reemergentes (Rouquayrol \& Silva, 2018) pela magnitude e extensão dos danos causados, necessitando de propostas de enfrentamento envolvendo os campos da saúde, educação, política, ciência, economia e relações sociais (Maia \& Reis Jr, 2019).

Dados epidemiológicos acerca do agravo o caracterizam como problema de saúde pública mundial (Almeida Filho \& Barreto, 2013). Na comparação dos dados epidemiológicos de 2005 e 2015, se observou um aumento de 16,9\% no total de pessoas vivendo com HIV/aids (PVHAs) no mundo, que passaram de 31,4 milhões para 36,7 (Brasil, 2017b).

No Brasil, em período semelhante, o acréscimo no número de casos foi de 193\%. O número passou de 463.992 em 2006 para 894.029 PVHAs em 2015 (Brasil, 2017b). Aqui cabe um importante destaque, pois o Boletim Epidemiológico Nacional aponta casos de pessoas vivendo com HIV somente a partir de 2007, conforme as normatizações de notificação do Ministério da Saúde. Em 2015, aproximadamente 15\% do total de casos notificados de pessoas vivendo com HIV/aids no Brasil são de adultos jovens, o que corresponde a 134.050 indivíduos de 20 a 29 anos (Brasil, 2017b).

Um outro dado que chama a atenção na epidemia em adultos jovens no país pode ser observado na comparação entre as taxas de detecção nos indivíduos do sexo masculino entre os anos de 2006 e 2016. Neste grupo, na faixa etária entre 20 e 24 anos houve acréscimo de 112\% de PVHAs, passando de 16,0 para 33,9/100 mil habitantes, enquanto na faixa entre 25 e 29 anos o acréscimo foi de $17 \%$, ou seja, de 41,1 para 48,3/100 mil habitantes. Diferentemente do identificado no grupo do sexo feminino, que na faixa de 20 a 24 anos teve queda de 25\%, diminuindo de 13,7 para 10,2/100 mil habitantes, e na faixa etária de 25 a 29 anos apresentou um declínio de 51\%, ou seja, de 30,5 para 15,0/100 mil habitantes (Brasil, 2017b).

No Paraná, até o ano de 2015, 44.460 pessoas viviam com HIV/aids, o que corresponde a aproximadamente $5 \%$ dos indivíduos notificados em todo o país naquele ano (Paraná, 2015).

Entendendo que saúde-doença é um processo dialético, no qual as dimensões individuais e coletivas devem ser analisadas no contexto em que são produzidas, a Epidemiologia não pode restringir seu olhar aos números que são expressões de expectativa de vida, nascimentos, adoecimentos e mortes na população, ao contrário, deve estudar as contradições da 
realidade que determinam as expressões de saúde e doença das populações em territórios históricos e socialmente construídos, visto que a realidade que observamos é determinada por processos que estão nas diferentes dimensões: geral, particular e singular. Assim, se faz necessário superar as abordagens empíricas sobre desigualdades sociais no sentido de buscar as explicações para as diferenças de acesso ao trabalho e renda, aos serviços de saúde e aos direitos sociais que determinadas sociedades lutam para garantir. Diferenças essas que ocorrem pelas relações de classe social, gênero, etnia/raça e geração presentes na sociedade e que se concretizam nas condições materiais de vida das populações e, consequentemente, nos seus perfis de saúde, doença e cuidado (Breilh, 2006).

Ao refletir sobre a superação de abordagens teóricas acerca das expressões individuais ou coletivas de saúde e doença, as quais ocorrem num determinado território, não se pode tomar esse espaço pela sua mera delimitação administrativa, física e geográfica, mas, sim, se deve compreendê-lo pelas relações históricas, sociais, culturais, econômicas, políticas e ambientais que se concretizaram naquele cotidiano. Entender aquela realidade espacial como um lugar onde saúde e doença, vida e morte pulsam, expressando as contradições e conflitos resultantes das relações de poder que ali se estabelecem pelos grupos hegemônicos e subalternos (Borde \& Torres-Tovar, 2017).

Com a epidemia de HIV, urge refletir acerca do modelo de atenção à saúde e do contexto que promove a desigualdade social, assim como não garante acesso aos bens e serviços públicos resolutivos em nossa sociedade. Nesta perspectiva, o presente estudo teve como objetivo discutir a epidemia do HIV em adultos jovens, de 20 a 29 anos, moradores dos municípios da Segunda Regional de Saúde do Paraná, no período 2007 a 2015, na perspectiva da Epidemiologia Crítica.

\section{Metodologia}

Trata-se de um estudo retrospectivo do tipo ecológico (Rouquayrol \& Silva, 2018), sob a ótica da Epidemiologia Crítica (Breilh, 2006). Para o desenvolvimento deste artigo seguiram-se os passos propostos pela Iniciativa Strengthening the Reporting of Observational Studies in Epidemiology (STROBE) (Elm et al., 2008), traduzida para a língua portuguesa por Malta et al. (2010).

O cenário da pesquisa foi a Segunda Regional de Saúde do Paraná ( $\left.2^{\mathrm{a}} \mathrm{RS}\right)$, que é composta por 29 municípios. Esta Regional corresponde à Região Metropolitana de Curitiba, onde está situada a capital do Estado do Paraná.

A amostra do estudo foi intencional, composta por jovens de 20 a 29 anos que viviam com HIV/Aids, notificados no SINAN-Net no período de 2007 a 2015, moradores de municípios da 2 ${ }^{\mathrm{a}}$ RS, que teve o número final de 2482 casos notificados.

A coleta de dados ocorreu em duas etapas. Na primeira etapa, entre os meses de abril a junho de 2017, foram elencados dados secundários disponíveis em sites públicos, na busca de evidenciar o modo de viver no território da $2^{\mathrm{a}} \mathrm{RS}$, entre eles: população censitária, tipo de moradia (urbana ou rural), indicadores sociais (Índice de Desenvolvimento Humano Municipal - IDH-M, Índice de Gini e Produto Interno Bruto - PIB), serviços de saúde, número de habitantes por raça/cor, taxa de analfabetismo, condições de infraestrutura (água encanada, luz elétrica, tratamento de esgoto), buscando apresentar dados referentes ao ano de 2015. Na segunda etapa os dados foram coletados do Sistema de Notificação de Agravos de Notificação versão para Internet (SINAN-Net), da Secretaria de Estado da Saúde do Paraná (SESA/PR), atualizado em 24 de abril de 2017. A coleta das informações junto ao setor responsável foi no período de junho a agosto de 2017.

A organização e tratamento dos dados de caráter qualitativo foram norteados pela compreensão acerca da caracterização do modo de viver na $2^{\mathrm{a}}$ RS. Os dados de caráter quantitativo, notificações de jovens de 20 a 29 anos que viviam com HIV/aids no período de 2007 a 2015, foram organizadas por meio do Microsoft Excel 2016®, no qual foi possível associar variáveis, calcular médias, percentuais e, ainda, desenvolver tabelas e gráficos. Entre as variáveis associadas no presente estudo, destacam-se: sexo, faixa etária, raça/cor, escolaridade, ocupação e local de moradia.

Tendo em vista que o estudo analisou um banco de dados fechado, restrito a variáveis coletadas na ficha individual de 
notificação (FIN), a análise do estudo com base nas categorias centrais da Epidemiologia Crítica - gênero, etnia e classe social - foi limitada, visto que as informações registradas na FIN são restritas a sexo, raça declarada e ocupação do indivíduo. Por isso, tomou-se como categoria central de análise o território, com o qual se buscou explorar o modo de viver dos indivíduos e, na sequência, relacionar esta caracterização aos dados epidemiológicos evidenciados.

Para aprofundar a discussão sob a ótica da Epidemiologia Crítica, definiu-se discutir com mais detalhes os dados dos dez municípios com mais de 50 casos ou prevalência maior ou igual a 0,4\%, sejam eles para HIV ou aids, ou, ainda, o somatório dos dois dados entre os 29 municípios da $2^{\text {a }}$ RS do Estado do Paraná.

O estudo foi aprovado pelo Comitê de Ética em Pesquisa da Universidade Federal do Paraná, sob o Parecer de ${ }^{\circ}$ 1.988.988, posteriormente, sua viabilidade e a coparticipação da Secretaria de Estado da Saúde do Paraná foram aprovadas pelo Comitê de Ética em Pesquisa do Hospital do Trabalhador/SESA, sob o Parecer n ${ }^{\circ}$ 2.035.675. Na submissão do projeto, foi solicitada a liberação do preenchimento do Termo de Consentimento Livre e Esclarecido, uma vez que a pesquisa coletou e analisou dados de fontes secundárias, nos quais não se teve acesso ao nome, endereço (logradouro) e nome da mãe dos indivíduos estudados, o que garantiu o anonimato das pessoas notificadas no sistema.

\section{Resultados}

O Paraná tem uma população de 11.163.018 habitantes distribuída em 399 municípios, apresenta densidade demográfica de 56 hab./km2. Na divisão sanitária o território possui 22 Regionais de Saúde (Paraná, 2017).

A $2^{\mathrm{a}} \mathrm{RS}$ é composta por 29 municípios, tem uma extensão territorial de $16.627,209 \mathrm{~km} 2$. A densidade demográfica média da Regional é de 348,64 hab./km2, com destaque para a alta concentração de habitantes em Curitiba, com 4.027,04 hab./km2, e a baixa concentração de habitantes em Adrianópolis, com 4,73 hab./km2, observadas na Tabela 1. (Paraná, 2017, Brasil, 2017c).

O total de habitantes em 2015 era de 3.223.796, tornando-a a regional mais populosa do estado. Destes, 48,7\% eram do sexo masculino e 51,3\% eram mulheres; acrescenta-se ainda que $18,2 \%$ do total de habitantes estavam na faixa de idade entre 20 e 29 anos (Brasil, 2017c) e a população da $2^{a}$ RS era predominantemente da raça/cor branca (74,5\%), seguida pela raça/cor parda $(21,3 \%)$, preta $(3,0 \%)$, amarela $(1,0 \%)$ e indígena $(0,1 \%)$. (Paraná, 2017).

A $2^{\mathrm{a}}$ RS possui 8.134 estabelecimentos de saúde, destes, 757 são do Sistema Único de Saúde (SUS), dos quais 14 são Serviços de Assistência Especializada (SAEs) e/ou Centro de Testagem e Aconselhamento (CTA) para HIV/Aids (Brasil, 2017d, Brasil, 2017e, Programa das Nações Unidas para o Desenvolvimento, 2013).

A capital do estado concentra $54,3 \%$ da população regional, $80,1 \%$ dos estabelecimentos de saúde, $30,2 \%$ dos estabelecimentos do SUS e 50\% dos SAEs e/ou CTA para HIV/Aids (Brasil, 2017d, Brasil, 2017e, Programa das Nações Unidas para o Desenvolvimento, 2013).

A Tabela 1 demonstra que a maior taxa de analfabetismo em maiores de 15 anos $(18,3 \%)$ e a menor renda familiar (1,7 salários mínimos) estão no Município de Tunas do Paraná, e no extremo oposto está Curitiba, que apresenta a menor taxa de analfabetismo em maiores de 15 anos (2,1\%) e a maior renda familiar (4,3 salários mínimos) (Brasil, 2017c, Brasil, 2017e).

No que tange às condições de infraestrutura dos municípios, se verificou que, na cidade de Cerro Azul, 5,9\% dos lares não têm água tratada, em Adrianópolis 4,9\% das residências não têm luz elétrica. Já Curitiba, Fazenda Rio Grande e Pinhais contam com $100 \%$ de cobertura de luz elétrica em seus domicílios. Com relação ao tratamento domiciliar de esgoto, os Municípios de Adrianópolis, Campo do Tenente, Doutor Ulysses, Piên, Rio Branco do Sul, Tijucas do Sul e Tunas do Paraná não apresentam registro desta infraestrutura, já os Municípios de Campina Grande do Sul, Curitiba e Pinhais têm cobertura acima de 100\%, e nas cidades de Agudos do Sul, Almirante Tamandaré, Balsa Nova, Bocaiúva do Sul, Campo Magro, Cerro Azul, Itaperuçu, Mandirituba, Quitandinha o total de domicílios atendidos é menor que 50\% (Paraná, 2017). 
No que diz respeito à coleta de lixo, o município com o menor percentual de domicílios atendidos é o de Doutor Ulysses, com 27,1\%. Somente a cidade de Pinhais tem $100 \%$ das residências atendidas, mas é importante destacar que, nas localidades de Almirante Tamandaré, Araucária, Campina Grande do Sul, Colombo, Curitiba, Fazenda Rio Grande, Piraquara e Quatro Barras, 95\% das casas são atendidas (Paraná, 2017).

Tabela 1 - Renda e analfabetismo em maiores de 15 anos, na Segunda Regional de Saúde do Paraná, Brasil, 2018.

\begin{tabular}{|c|c|c|c|}
\hline & & \multirow{2}{*}{$\begin{array}{l}\text { RENDA (2014) } \\
\text { salários mínimos } \\
\end{array}$} & \multirow{2}{*}{$\begin{array}{c}\text { ANAFALBETISMO } \\
>\text { > 15 A } \\
\text { Estado } 6,28\end{array}$} \\
\hline & & & \\
\hline \multirow{29}{*}{ 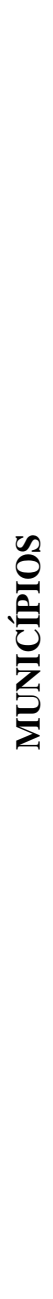 } & Adrianópolis & 3,1 & 16,82 \\
\hline & Agudos do Sul & 1,7 & 8,84 \\
\hline & Almirante Tamandaré & 2,2 & 6,06 \\
\hline & Araucária & 4,3 & 3,55 \\
\hline & Balsa Nova & 3,0 & 4,55 \\
\hline & Bocaiúva do Sul & 2,0 & 9,05 \\
\hline & Campina Grande do Sul & 2,5 & 6,03 \\
\hline & Campo do Tenente & 1,9 & 9,06 \\
\hline & Campo Largo & 2,6 & 4,50 \\
\hline & Campo Magro & 2,8 & 6,17 \\
\hline & Cerro Azul & 1,7 & 17,73 \\
\hline & Colombo & 2,3 & 4,65 \\
\hline & Contenda & 2,3 & 5,06 \\
\hline & Curitiba & 4,1 & 2,13 \\
\hline & Doutor Ulysses & 1,8 & 17,34 \\
\hline & Fazenda Rio Grande & 2,5 & 4,47 \\
\hline & Itaperuçú & 1,8 & 10,79 \\
\hline & Lapa & 2,3 & 5,67 \\
\hline & Mandirituba & 2,0 & 6,63 \\
\hline & Piên & 2,9 & 4,21 \\
\hline & Pinhais & 2,7 & 3,35 \\
\hline & Piraquara & 2,4 & 5,03 \\
\hline & Quatro Barras & 3,2 & 4,73 \\
\hline & Quitandinha & 2,1 & 7,11 \\
\hline & Rio Branco do Sul & 2,7 & 10,47 \\
\hline & Rio Negro & 2,2 & 3,54 \\
\hline & São José dos Pinhais & 3,6 & 3,40 \\
\hline & Tijucas do Sul & 2,6 & 8,59 \\
\hline & Tunas do Paraná & 1,7 & 18,25 \\
\hline
\end{tabular}

A $2^{\text {a }}$ RS detém o pior e o melhor IDH-M do estado, sendo o pior índice na cidade de Doutor Ulysses, com o IDH-M de 0,546, e o melhor indicador em Curitiba, com o IDH-M de 0,823. O Índice de Gini apresenta discrepâncias entre os municípios, a maior desigualdade de renda $(0,5652)$ está representada na capital e a menor desigualdade $(0,3984)$ está no Município de Campo Magro. A cidade com o maior PIB per capita é São José dos Pinhais (R \$79.268), polo industrial e território municipal adjacente a Curitiba, enquanto o menor é PIB está no Município de Doutor Ulysses (R\$ 10.560), que tem sua caracterização como território rural (Paraná, 2017).

O total de casos de HIV/aids na $2^{\text {a }}$ RS, no período de 2007 a 2015, foi de 2.482 indivíduos de 20 a 29 anos. Destaca-se que, destes, 2.376 (95,7\%) são de notificações de HIV e 106 (4,3\%), de notificações de aids. 
Quando os casos são desmembrados em duas faixas etárias, observa-se que, nos indivíduos entre 20 e 24 anos, são $1.110(44,7 \%)$ notificações de HIV e 39 (1,6\%) notificações de aids, enquanto na faixa de 25 a 29 anos são $1.266(51,0 \%)$ casos de HIV e 69 (2,7\%) casos de aids, conforme se observa detalhadamente no Gráfico 1.

Gráfico 1 - Distribuição dos casos de HIV e aids, segundo a faixa etária, na Segunda Regional de Saúde do Paraná, Brasil, 2018

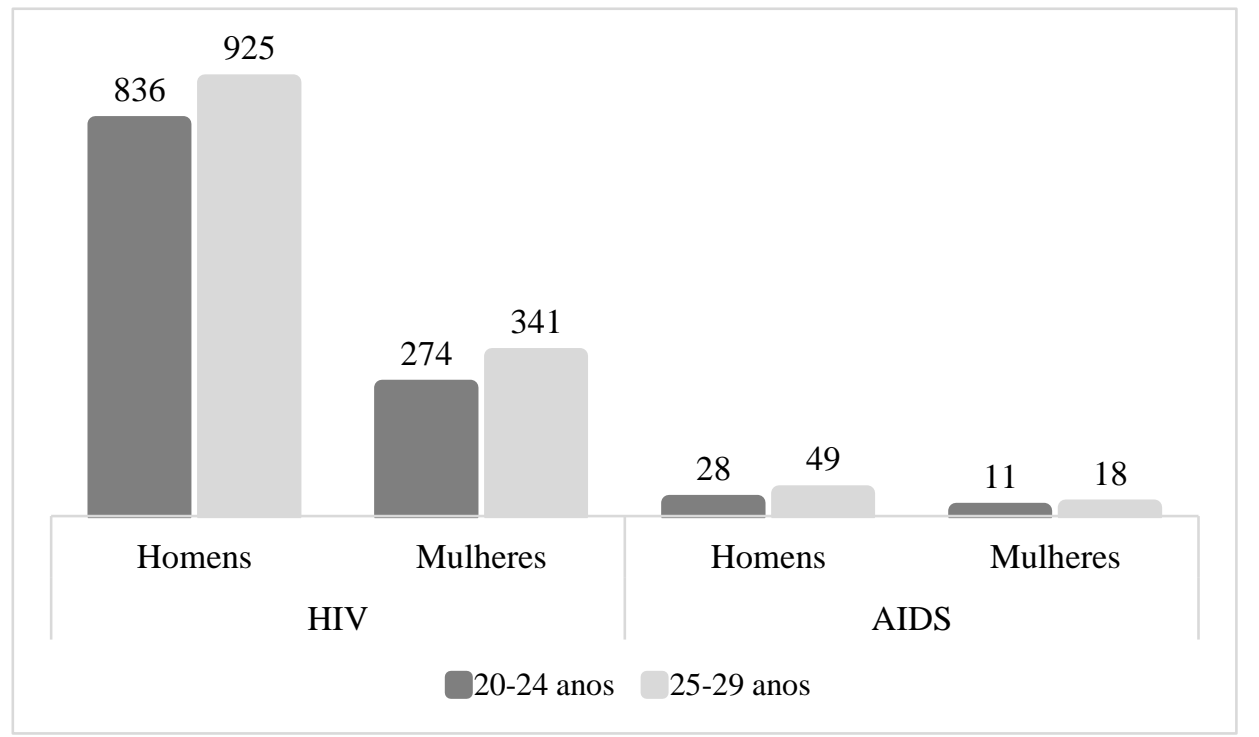

Legenda: Aids - Síndrome da Imunodeficiência Adquirida; HIV - Vírus da Imunodeficiência Humana. Fonte: Autores

No que concerne à raça/cor autodeclarada, $1.855(74,7 \%)$ pessoas eram da raça/cor branca, seguidas por $312(12,6 \%)$ indivíduos da raça/cor parda e 107 (4,3\%) da raça/cor preta; nas demais notificações, 23 (0,9\%) autodeclararam-se da raça/cor amarela ou indígena e 185 (7,5\%) tiveram esse campo da FIN não preenchido ou ignorado.

As notificações demonstraram diferenças na escolaridade entre os casos de HIV e aids, uma vez que, nos casos de HIV, 489 (20,6\%) dos indivíduos tinham ensino médio completo. Nas notificações pela síndrome, houve predomínio do ensino fundamental incompleto, com $32(30,2 \%)$ dos casos. Esta variável apresentou 710 casos (28,6\%) com o campo ignorado ou não preenchido na FIN. A Tabela 2 demonstra detalhadamente o número de casos conforme a escolaridade. 
Tabela 2 - Notificações de HIV e aids, conforme a escolaridade, na Segunda Regional de Saúde do Paraná, Brasil, 2018

\begin{tabular}{lcc} 
& HIV & AIDS \\
\cline { 2 - 3 } & $\mathrm{n}(\%)$ & $\mathrm{n}(\%)$ \\
\hline Analfabeto & $2(0,1 \%)$ & $0(0,0 \%)$ \\
$1^{\mathrm{a}}$ a $4^{\mathrm{a}}$ incompleta & $56(2,4 \%)$ & $5(4,7 \%)$ \\
$4^{\mathrm{a}}$ completa & $70(2,9 \%)$ & $5(4,7 \%)$ \\
$5^{\mathrm{a}}$ a $8^{\mathrm{a}}$ incompleta & $247(10,4 \%)$ & $22(20,8 \%)$ \\
Ensino Fundamental & $188(7,9 \%)$ & $12(11,3 \%)$ \\
Ensino Médio Incompleto & $198(8,3 \%)$ & $10(9,4 \%)$ \\
Ensino Médio & $489(20,6 \%)$ & $14(13,2 \%)$ \\
Ensino Superior Incompleto & $194(8,2 \%)$ & $1(0,9 \%)$ \\
Ensino Superior & $253(10,6 \%)$ & $6(5,7 \%)$ \\
Ignorado & $604(25,4 \%)$ & $28(26,4 \%)$ \\
Não preenchido & $75(3,2 \%)$ & $3(2,8 \%)$ \\
Não se aplica & $0(0,0 \%)$ & $0(0,0 \%)$ \\
\hline TOTAL & $2376(100 \%)$ & $106(100 \%)$ \\
\hline Aids - Síndrome da Imunodeficiência & Adquirida; HIV & - Vírus da \\
Imunodeficiência Humana. Fonte: Autores. & &
\end{tabular}

Outra característica importante é a ocupação do indivíduo na FIN, onde 669 (26,9\%) tiveram a ocupação preenchida conforme a Classificação Brasileira de Ocupações (CBO) (Brasil, 2010). Entre as principais, estão: trabalhadores dos serviços, vendedores do comércio em lojas e mercados nos casos de HIV; e trabalhadores das ciências e das artes e trabalhadores da produção de bens e serviços indústria nas notificações pela síndrome. As ocupações codificadas para fins de cadastro no SINAN-Net e que não são contempladas na CBO, com maior percentual de casos, são: donas de casa com 141 (5,7\%) notificações e desempregados com 88 (3,5\%). Este campo apresentou registro ignorado ou não preenchido em 1.468 (59,1\%) notificações de HIV/Aids, na amostra estudada.

Quanto ao local de moradia dos indivíduos, 2.381 (95,9\%) moravam na zona urbana; 62 (2,5\%), na zona rural; dois $(0,1 \%)$, na zona periurbana; e $37(1,5 \%)$ das notificações tiveram este campo ignorado ou não preenchido.

Com relação ao local de registro da FIN, observou-se que 839 casos (33,8\%) foram notificados no município de moradia do indivíduo, enquanto 404 (16,3\%), no município sede, que no caso é a capital do estado, ao passo que a maioria das notificações, 1.239 (49,9\%), foi notificada em outros municípios. Acrescenta-se aqui que somente Curitiba, Pinhais e São José dos Pinhais notificaram seus munícipes. Os demais casos foram notificados por serviços de saúde que não pertenciam ao município de moradia da pessoa notificada com HIV.

A Tabela 2 demonstra os dez municípios nos quais buscou-se reconhecer a determinação social do fenômeno HIV. Nestes municípios a população é majoritariamente residente na zona urbana, com exceção de Piraquara. Os dados apontam que nestas cidades há a mesma proporção de indivíduos por raça/cor, taxa de analfabetismo na população de adultos jovens, entre 0,52\% e 1,58\%. Aproximadamente $98 \%$ dos domicílios desses municípios têm água canalizada, banheiro, energia elétrica e coleta de lixo, à exceção de Almirante Tamandaré e Araucária, que têm 47,3\% e 79,1\% de residências com cobertura de tratamento de esgoto, respectivamente. 
Tabela 3 - Índices socioeconômicos e taxas de HIV/aids nos municípios da Segunda Regional de Saúde do Paraná, Brasil, $2018(\mathrm{n}=2.373)$.

\begin{tabular}{|c|c|c|c|c|c|c|c|}
\hline \multirow{2}{*}{ Município } & \multirow{2}{*}{ IDH-M } & \multirow{2}{*}{ Índice Gini } & \multirow{2}{*}{ PIB } & \multicolumn{2}{|c|}{ HIV } & \multicolumn{2}{|c|}{ Aids } \\
\hline & & & & $\mathbf{n}$ & Prev.* & $\mathbf{n}$ & Prev.* \\
\hline Almirante Tamandaré & 0,699 & 0,4402 & 11.375 & 65 & $0,4 \%$ & 1 & $0,0 \%$ \\
\hline Araucária & 0,740 & 0,4637 & 65.153 & 62 & $0,3 \%$ & 6 & $0,0 \%$ \\
\hline Campo Magro & 0,701 & 0,3984 & 10.653 & 16 & $0,4 \%$ & 0 & $0,0 \%$ \\
\hline Colombo & 0,733 & 0,4196 & 18.963 & 104 & $0,3 \%$ & 10 & $0,0 \%$ \\
\hline Curitiba & 0,823 & 0,5652 & 42.315 & 1670 & $0,5 \%$ & 40 & $0,0 \%$ \\
\hline Fazenda Rio Grande & 0,720 & 0,4922 & 18.641 & 56 & $0,4 \%$ & 5 & $0,0 \%$ \\
\hline Pinhais & 0,751 & 0,5082 & 40.994 & 122 & $0,6 \%$ & 29 & $0,1 \%$ \\
\hline Piraquara & 0,700 & 0,4307 & 10.679 & 60 & $0,3 \%$ & 3 & $0,0 \%$ \\
\hline São José dos Pinhais & 0,758 & 0,4599 & 79.268 & 117 & $0,2 \%$ & 2 & $0,0 \%$ \\
\hline Tunas do Paraná & 0,611 & 0,5283 & 15.112 & 5 & $0,4 \%$ & 0 & $0,0 \%$ \\
\hline TOTAL & - & - & - & 2277 & $0,4 \%$ & 96 & $0,0 \%$ \\
\hline $2^{\mathrm{a}} \mathrm{RS}$ & 0,783 & 0,5629 & 38.872 & 2376 & $0,4 \%$ & 106 & $0,02 \%$ \\
\hline
\end{tabular}

Legenda: Aids - Síndrome da Imunodeficiência Adquirida; HIV - Vírus da Imunodeficiência Humana; IDH-M - Índice de Desenvolvimento Humano - Municipal; PIB - Produto Interno Bruto; Prev. - prevalência; $2^{\mathrm{a}} \mathrm{RS}$ - Segunda Regional de Saúde Nota: *taxa calculada com base na população de 20 a 29 anos do município. Fonte: Autores.

\section{Discussão}

A maior densidade populacional no entorno de Curitiba converge com achados do estudo de Cardoso (2015), realizado na Europa, o qual demonstra que as cidades no entorno dos municípios sedes e das capitais são mais densas pela possibilidade de inserção dos indivíduos no trabalho e, consequentemente, acesso às condições melhores para a vida. Dinâmica que está essencialmente relacionada ao desenvolvimento econômico dos municípios da $2^{\mathrm{a}} \mathrm{RS}$, principalmente aqueles que estão em territórios adjacentes ao município sede, pois historicamente as populações de menor poder aquisitivo buscam morar em áreas com o solo menos valorizado, mas com fácil acesso à capital, na perspectiva de se inserirem nos processos formais de produção, formação pessoal e profissional (Paraná, 2017).

A $2^{\mathrm{a}}$ RS tem a pior cobertura de serviços de saúde que atendem PVHAs da Região Sul, aproximadamente um serviço para cada 230.274 habitantes. Relação inferior à de serviços ofertados nos municípios que estão no entorno de Porto Alegre e Florianópolis: um para cada 149.321 habitantes e um para cada 125.301 habitantes, respectivamente (Brasil, 2017c, Programa das Nações Unidas para o Desenvolvimento, 2013). Estes serviços são compreendidos como essenciais para o diagnóstico e aconselhamento em HIV e aids, pois acredita-se que o acesso aos serviços especializados leve ao diagnóstico precoce, o que leva a um melhor prognóstico no tratamento.

Entre as capitais e suas regiões metropolitanas do Sul do país, o território da $2^{\mathrm{a}} \mathrm{RS}$ ocupa o $5^{\mathrm{o}}$ lugar no ranking nacional do IDH-M, enquanto Florianópolis ocupa o $1^{\circ}$ lugar e Porto Alegre, o $13^{\circ}$ lugar (Programa das Nações Unidas para o Desenvolvimento, 2013). Partindo do pressuposto de que, quanto melhor este indicador, melhores são as condições de educação, saúde e renda, morar em cidades que tenham alto IDH-M beneficia o cuidado com a saúde, bem como favorece a busca por melhores condições de vida, trabalho e moradia, processos considerados como protetores da saúde (Breilh, 2006). Tal premissa permite refletir acerca dos índices que indicam o crescimento de PVHAs, pois é significativo o aumento de adultos jovens vivendo com HIV/Aids no território e período estudado. 
A disparidade entre o índice de Gini nos municípios da $2^{a}$ RS também é observada em municípios de outras regiões metropolitanas brasileiras, como, por exemplo, São Paulo, Florianópolis e Porto Alegre (Programa das Nações Unidas para o Desenvolvimento, 2013). A contradição que se verifica no índice de Gini no Brasil, nos últimos anos, é que está em queda, um fenômeno que representa a diminuição da desigualdade socioeconômica na sociedade. Porém, a renda da população moradora de regiões mais pobres do Brasil continua próximo de 0,4 a 1,6 salários mínimos, enquanto a renda dos moradores das regiões metropolitanas varia entre 1,3 e 3,2 salários mínimos, o que reafirma a expressão de desigualdade social no país (Montali \& Lessa, 2016).

Quanto ao PIB, observa-se que há discrepância entre os municípios da $2^{\mathrm{a}} \mathrm{RS}$, assim como nas regiões adstritas a Porto Alegre e São Paulo (Brasil, 2017c). A concentração de riquezas na cidade de São José dos Pinhais pode estar relacionada ao fato de esta ser polo industrial da região, enquanto a cidade de Doutor Ulysses ainda se caracteriza mais como território rural. É importante registrar que há limitação neste índice, pois o PIB é uma medida agregada e, como tal, não pode informar sobre a distribuição da riqueza entre a população, mas indica o quanto de riqueza se produz em determinado território naquele período (Eurostat Statistics Explained, 2018).

O achado sobre a raça/cor na $2^{\mathrm{a}}$ RS diverge dos dados censitários nacionais, nos quais $47,7 \%$ dos brasileiros se declararam brancos, 43,1\%, pardos e 7,6\%, negros, mas assemelha-se à realidade da Região Sul, onde se declararam brancos $84 \%$ da população catarinense e $83,4 \%$ da população gaúcha (Brasil, 2017c). Assim como confirma os achados de um estudo desenvolvido na $10^{\mathrm{a}}$ RS do Paraná, região oeste do estado, que considerou a raça/cor autodeclarada na FIN e identificou que 67,4\% das notificações de HIV/Aids eram de indivíduos da raça/cor branca, seguidos por 29,3\% dos de raça/cor parda (Silva et al., 2017).

Com relação às taxas de analfabetismo na $2^{\mathrm{a}} \mathrm{RS}$, há grande dissonância e variação entre a população moradora de seus municípios. Tunas do Paraná tem taxa semelhante aos municípios da Região Nordeste (18,5\%), ou seja, assemelha-se às piores taxas de analfabetismo do país, e Curitiba tem taxa abaixo da média da Região Sul (4,5\%), entre as melhores do país (Brasil, 2017e). Estes dados corroboram a reflexão sobre as expressões de desigualdade social no território da $2^{\text {a }}$ RS, evidenciada por este estudo.

Face ao número de casos de HIV e aids, a amostra estudada divergiu da realidade nacional, na qual a população de 2029 anos totalizou 141.727 casos no período de 2007-2015, sendo 31,5\% casos de HIV e 58,6\% de aids (Brasil, 2017b). Da mesma forma, no Estado do Rio Grande do Sul, os casos notificados como HIV representaram 16,5\% e os de aids, 83,5\% (Rio Grande do Sul, 2017). Destarte, observa-se maior sensibilidade para notificação de HIV na $2^{\mathrm{a}}$ Regional de Saúde, dado que pode estar relacionado ao aumento de cobertura de serviços da atenção básica à saúde na regional ou ao fato de Curitiba notificar os casos de HIV desde 2002 (Curitiba, 2014), vislumbrando, assim, um achado que precisa ser explorado com mais profundidade, visto que nem todos os casos do agravo foram notificados pela capital, conforme demonstrado na Tabela 3.

Na subdivisão por faixas etárias dos jovens, a amostra do presente estudo diverge da amostra nacional (Brasil, 2017b), uma vez que, na amostra nacional, a faixa etária de 20 a 24 anos perfaz 39,6\% do total de casos, enquanto na faixa de 25 a 29 anos, $60,4 \%$ do total de casos adultos é de jovens que viviam com HIV/Aids. Assim como também difere no total de casos notificados por HIV e daqueles notificados por aids, na amostra nacional, o total de casos de HIV é de 37,5\% e o total de casos de aids é de 62,5\% (Brasil, 2017b). Convergente com os dados do presente estudo, pois os indivíduos entre 25 e 29 anos apresentam o maior número de casos, tanto no Paraná, quanto na $2^{\mathrm{a}} \mathrm{RS}, 2115$ (52\%) e 1350 (54\%), respectivamente.

Relativamente à escolaridade da população estudada, observaram-se diferenças entre aqueles notificados por HIV e aqueles notificados pela síndrome. Um estudo brasileiro verificou que a maioria dos indivíduos com HIV (27,9\%) tinha ensino fundamental incompleto (Dartora et al., 2017) e fundamental completo (Dietz et al., 2021), enquanto, nos casos de aids, a amostra desta pesquisa assemelha-se ao encontrado em estudo realizado no Estado do Maranhão (Galvão et al., 2017), no qual 
a maioria das notificações (40\%) de aids informava ensino fundamental incompleto. Conforme os preceitos da Epidemiologia Crítica, a escolaridade pode ser considerada um processo protetivo e favorecedor das defesas e suportes, e favorável à vida humana individual e coletiva (Breilh, 2006). Nesta pesquisa verificamos maior escolaridade entre os casos notificados com HIV, em relação aos casos notificados por aids, o que indica um diagnóstico precoce do agravo em pessoas com maior nível de instrução.

$\mathrm{Na}$ conclusão de uma pesquisa realizada em Botsuana, África Austral, a escolaridade foi vista como protetora na contaminação pelo HIV, pois investimentos em educação se tornaram uma medida econômica de prevenção ao vírus, essencialmente para as mulheres (De Neve et al., 2015). Neste sentido, a identificação e, consequentemente, o preenchimento do campo referente à variável na notificação é imprescindível (Canto \& Nedel, 2020), para que, assim, se evidenciem os níveis de escolaridade da população atingida, a fim de que se elaborem políticas públicas que objetivem diminuir os processos de iniquidades relacionados a estas questões (Breilh, 2006, De Neve et al., 2015).

Referente à ocupação do indivíduo, embora a Portaria MS no 3.497, de 25 novembro de 1998, defina que a ocupação na FIN deva ser preenchida de acordo com a CBO, poucos estudos apontam os resultados dessa maneira, ao contrário, os dados são categorizados de outras formas, tais como: ocupado ou não, sem categorização específica (São Paulo, 2016, Meirelles et al., 2016), inclusive com perfis do agravo que sequer contemplam a ocupação do indivíduo (Brasil, 2017b, Silva et al., 2017, Rio Grande do Sul, 2017). Quanto ao alto percentual de notificações que tiveram esse campo ignorado ou não preenchido, este estudo assemelha-se a outros realizados no Rio Grande do Norte e em São Paulo, com, respectivamente, 51\% e 49\% de dados relacionados a esta variável não preenchida (São Paulo, 2016, Meirelles et al, 2016). As dificuldades de seguir os extensos parâmetros da classificação, assim como a diversidade de profissões atualmente registradas podem ser o principal motivo do não preenchimento do campo. A forma de inserção nos modos de produção é considerada um processo determinante para o padrão de vida e o modo de viver das pessoas e seus dependentes, o que gera processos protetivos ou destrutivos que podem ocasionar mudanças significativas na saúde dos coletivos e indivíduos (Breilh, 2006).Considera-se fundamental elaborar estudos sobre as ocupações daqueles que são mais acometidos pelo agravo, para que se possa implementar medidas que possibilitem mudanças mais efetivas nas formas de adoecer e morrer dessas pessoas.

Referente ao local de moradia, tanto este estudo como outros apontam que o HIV tem características urbanas, essencialmente em regiões metropolitanas (Rio Grande do Sul, 2017, São Paulo, 2016, Limas et al., 2021). O que se justifica, pois as doenças infecciosas com transmissão que não envolvem vetores se espalham relativamente mais rápido em uma aglomeração mais densa de pessoas, possivelmente, porque entre essas há um maior número de contatos (Rocha et al., 2015).

Atualmente é possível verificar e associar o fenômeno HIV à baixa renda (Lopes et al., 2015) e à baixa escolaridade (Dietz et al., 2021) a que estão submetidas as populações mais acometidas, o que é uma realidade que se observa nas sociedades, nas quais é a estrutura econômica, social e política que define os processos de proteção e de desgastes a que estão expostos os indivíduos, os quais se reproduzem mediante a sua inserção no trabalho e as possíveis condições de vida naquele território (Breilh, 2006, Borde \& Torres-Tovar, 2017).

O custo baixo ou custo zero com o deslocamento das pessoas para acessar serviço de saúde se mostrou favorável ao diagnóstico de HIV em um estudo realizado em Porto Alegre (Zambenedetti \& Silva, 2015), em geoprocessamento realizado no Município de Londrina, Paraná (Pieri et al., 2021), apontou adensamento dos casos de HIV e aids nas proximidades do CTA, contraditoriamente ao encontrado neste estudo. Para Grangeiro (2016), que avaliou os quarenta anos da aids no Brasil, há ineficiência de respostas às questões estruturais, tais como discriminação e preconceito, tão presentes nos cotidianos das PVHAs, assim como há necessidade de fazer a correção no déficit de profissionais, nas estratégias de acolhimento e na vinculação de usuários nos serviços de saúde. Pois esses processos, provavelmente, são desencadeadores do deslocamento do indivíduo na busca dos serviços de saúde mais distantes para o diagnóstico. 
No Rio Grande do Sul, um estudo indicou que a qualidade da assistência prestada pela atenção primária a crianças e adolescentes vivendo com HIV tem sido satisfatória (Piovesan et al., 2017), ou seja, há potencialidade nos serviços locais para o enfrentamento deste fenômeno. Portanto, não se pode pactuar com a ausência de estratégias que visem combater desigualdades, preconceitos, precariedades e discriminações, claramente evidenciados no fenômeno HIV em uma sociedade (Maia, \& Reis Jr, 2019).

\section{Considerações Finais}

O fenômeno estudado, a epidemia de HIV em adultos jovens de uma Regional de Saúde, sob a ótica da Epidemiologia Crítica, foi um grande desafio, uma vez que se pôde verificar que o processo saúde dos jovens que vivem com HIV está relacionado ao modo de viver em seus municípios de moradia, logo, às suas condições de vida. Tal afirmação se referenda nos índices apresentados nos dados dos municípios da $2^{\mathrm{a}} \mathrm{RS}$, pois, conforme demonstrado, os territórios que detêm as maiores taxas de prevalência são aqueles que têm a maior desigualdade de renda, ao passo que o maior número de notificações ocorreu em cidades que possuem os melhores IDH-M, PIB e organização dos serviços de saúde nos seus espaços, enquanto que os municípios com as piores taxas de analfabetismo, e essencialmente rurais, são os que possuem o menor número de notificações. Porém, é importante destacar a inexistência de limite territorial para as PVHAs, pois, na busca pelo anonimato no que tange ao enfrentamento e ao diagnóstico do agravo e do preconceito, a maioria dos jovens que viviam com HIV na $2^{\text {a }}$ RS no período de 2007-2015 foram buscar serviços de saúde em outros municípios que não o de sua moradia, e ali foram notificados.

Foi evidenciado, no cotidiano das pessoas que vivem nos municípios da regional cenário do estudo, que desigualdade de renda, dificuldade de acesso aos serviços de saúde e baixa escolaridade estão relacionados diretamente aos resultados encontrados, uma vez que a determinação social do processo saúde tem seu maior peso em processos vinculados à dimensão estrutural da sociedade, pois são esses que definem, em primeira instância, o modo de viver da população, pela inserção desta na produção e, consequentemente, o acesso ao trabalho e às condições materiais para a vida daí derivadas.

Refletir e apontar a determinação social do fenômeno foi a limitação deste estudo, uma vez que se utilizou banco de dados com variáveis pré-estabelecidas, impossibilitando contemplar as categorias gênero, etnia e classe social. Contraditoriamente, esta limitação estimulou a compreensão do território e do modo de viver dos indivíduos como categoria de análise.

Por fim, com os resultados do presente estudo afirmamos que cabe aprofundar, por meio de novas pesquisas, reflexões acerca dos indicadores econômicos das sociedades e dos índices epidemiológicos, comportamentos individuais e coletivos presentes no fenômeno HIV, no sentido de elaborar ações intersetoriais para o seu enfrentamento.

\section{Agradecimentos}

À Coordenação de Aperfeiçoamento de Pessoal do Nível Superior, pela Bolsa Social, concedida à autora correspondente, durante o mestrado.

\section{Referências}

Borde, E. \& Torres-Tovar, M. (2017). El territorio como categoría fundamental para el campo de la salud pública. Saúde em Debate, 41(2), 264-75. https://doi.org/10.1590/0103-11042017S222

Brasil, Ministério do Trabalho e Emprego. (2010). Classificação Brasileira de Ocupações. Ministério do Trabalho e Emprego.

Brasil, Ministério da Saúde. (2017a). Cuidado integral às pessoas que vivem com HIV pela Atenção Básica: manual para a equipe multiprofissional. Brasília: Ministério da Saúde., https://bvsms.saude.gov.br/bvs/publicacoes/cuidado_integral_hiv_manual_multiprofissional.pdf 
Brasil, Ministério da Saúde. (2017b). Boletim Epidemiológico HIV Aids. Brasília: Ministério da Saúde. http://www.aids.gov.br/pt-br/pub/2017/boletimepidemiologico-hivaids-2017

Brasil, Instituto Brasileiro de Geografia e Estatística. (2017c). IBGE. https://www.ibge.gov.br/

Brasil, Ministério da Saúde. (2017d). Departamento de Vigilância, Prevenção e Controle das IST, do HIV/Aids e das Hepatites Virais. http://www.aids.gov.br/ Brasil, Ministério da Saúde. (2017e). Departamento de Informática do Sistema Único de Saúde. http://datasus.saude.gov.br/

Breilh, J. (2006). Epidemiologia Crítica: ciência emancipadora e interculturalidade. Fiocruz.

Canto, V.B. do, \& Nedel, F.B. Completude dos registros de tuberculose no Sistema de Informação de Agravos de Notificação (Sinan) em Santa Catarina, Brasil, 2007-2016. Epidemiol. Serv. Saúde, 29(3), e2019606. https://doi.org/10.5123/S1679-49742020000300020.

Cardoso, R. (2015). Cidades principais e secundárias na Europa: uma caracterização dos contrastes à escala da região urbana. J Geografic Spatial Plann. (7), 85-109. http://dx.doi.org/10.17127/got/2015.7.004

Curitiba, Secretaria Municipal de Saúde. (2014). Boletim Epidemiológico: Curitiba. Coordenação Municipal de DST/HIV/AIDS/Hepatites Virais.

Dartora, W. J., Ânflor, É. P., \& Silveira, L. R. P. da. (2017). Prevalência do HIV no Brasil 2005-2015: dados do Sistema Único de Saúde. Rev Cuid., 8(3), 1919-28. https://doi.org/10.15649/cuidarte.v8i3.462

De Neve, J-W., Günther, F., Subramanian, S. V., Moyo, S., \& Bor, J. (2015). Length of secondary schooling and risk of HIV infection in Botswana: evidence from a natural experiment. Lancet Glob Health, 3(8), e470-7. https://doi.org/10.1016/S2214-109X(15)00087-X

Dietz, J. do C., Borges, M. S., Oliveira, D. de L., Gomes, J. G., \& Manrique, E. J. C. Adolescentes e adultas jovens e a transmissão materno-infantil do HIV. (2021). Research, Society and Development, 10(1), e20410111440. https://doi.org/10.33448/rsd-v10i1.11440

Elm, E. von, Altman, D. G., Egger, M, Pocock, S. J., Gøtzsche, P. C. \& Vandenbroucke, J. P. (2008). STROBE Initiative. The Strengthening the Reporting of Observational Studies in Epidemiology (STROBE)statement: guidelines for reporting observational studies. J Clin Epidemiol, 61(4), 344-9. https://doi.org/10.1016/j.jclinepi.2007.11.008

Eurostat Statistics Explained. (2018, julho). Quality of life indicators - measuring quality of life. The European Union. http://ec.europa.eu/eurostat/statisticsexplained/index.php/Quality_of_life_indicators_-_measuring_quality_of_life

Galvão, J. M. V., Costa, A. C. M., \& Galvão, J. V. (2017). Demographic and socio-demographic profile of people living with HIV / AIDS. Rev Enferm UFPI., 6(1), 4-8. https://doi.org/10.26694/reufpi.v6i1.5533

Grangeiro, A. (2016). Da estabilização à reemergência: os desafios para o enfrentamento da epidemia de HIV / AIDS no Brasil nesta sua quarta década. In Basthi, A., Parker, R \& Terto Jr., V (Eds), Mito vs Realidade: sobre a resposta brasileira à epidemia de HIV e AIDS em 2016 (16-21). Associação Brasileira Interdisciplinar de AIDS.

Limas, F. M., Brandão, M. L., Luccas, D. S. de, Bossle, R. C., Khalaf, D. K. \& Chaves, M. M. N. (2021). Spatial analysis of HIV cases in young adults and access to public services in a municipality of Paraná. O Mundo da Saúde, 45(1), 337-347. doi: 10.15343/0104-7809.202145337347

Lopes, E. M., Pedrosa, N. L., Holanda, E. R., Almeida, R. L. F., Kerr, L. R. F. S., \& Galvão, M. T. G. (2015). AIDS em crianças: a influência dos diferenciais socioeconômicos no Município de Fortaleza, Ceará, Brasil. Cad Saúde Pública, 31(9), 2005-16. https://doi.org/10.1590/0102-311X00074514

Maia, E. C. A., \& Reis Jr, L. P. (2019). Modos de enfrentamento do HIV/AIDS: direitos humanos, vulnerabilidades e assistência à saúde. Rev. NUFEN, 11(1), 178-193. http://pepsic.bvsalud.org/pdf/rnufen/v11n1/a12.pdf

Malta, M., Cardoso, L. O., Bastos, F. I., Magnanini, M. M. F. \& Silva, C. M. F. P. (2010). Iniciativa STROBE: subsídios para a comunicação de estudos observacionais. Rev Saúde Pública, 44(3), 559-65. https://doi.org/10.1590/S0034-89102010000300021

Meirelles, M. Q. B., Lopes, A. K. B., \& Lima, K. C. (2016). Vigilância epidemiológica de HIV/Aids em gestantes: uma avaliação acerca da qualidade da informação disponível. Rev Panam Salud Publica, 40(6), 427-34. https://iris.paho.org/handle/10665.2/33662

Montali, L. \& Lessa, L. H. (2016). Pobreza e mobilidade de renda nas regiões metropolitanas brasileiras. Cad Metrópole, 18(36), 503-33. https://doi.org/10.1590/2236-9996.2016-3610

Paraná, Secretaria de Estado da Saúde. (2015). Boletim Epidemiológico HIV AIDS 2015. Secretaria de Estado da Saúde.

Paraná, Instituto Paranaense de Desenvolvimento Econômico e Social. (2017). IPARDES. http://www.ipardes.gov.br/

Pieri, F. M., Gonçalves, L. F. R., Gioia, T. B., Arroyo, L. H., Montanha, R. M. \& Ferreira, N. M. A., et al. (2021). HIV/AIDS: estudo ecológico de variações espaciais nas tendências temporais nos anos de 2007 a 2018. Research, Society and Development, 10(3), e5901031341. http://dx.doi.org/10.33448/rsdv10i3.13415.

Piovesan, G., Paula, C. C. de, Lopes, L. F. D., Padoin, S. M. de M., Kleinubing, R. E., \& Silva, C. B. da. (2017). Primary care quality from professional's perspective: health of children and adolescents with hiv. Texto contexto - enferm, 26(2), e00180016. https://doi.org/10.1590/0104-07072017000180016

Programa das Nações Unidas para o Desenvolvimento, Fundação João Pinheiro, Instituto de Pesquisa Econômica Aplicada. (2013). Atlas do Desenvolvimento Humano no Brasil. http://www.atlasbrasil.org.br/2013

Rio Grande do Sul, Secretaria de Estado da Saúde, Escola de Saúde Pública. (2017). Boletim Epidemiológico HIV/AIDS 2016. Porto Alegre: Secretaria de Estado da Saúde. 
Research, Society and Development, v. 11, n. 1, e9511124929, 2022

(CC BY 4.0) | ISSN 2525-3409 | DOI: http://dx.doi.org/10.33448/rsd-v11i1.24929

Rocha, L. E. C., Thorson, A. E., \& Renaud, L. The non-linear health consequences of living in larger cities. (2015). J Urban Health, 92(5), 784-99. https://doi.org/10.1007/s11524-015-9976-x

Rouquayrol, M. Z., \& Silva, M. G. C. da (2018). Rouquayrol - Epidemiologia e saúde. (8a ed.), MedBook.

São Paulo, Secretaria de Estado da Saúde. (2016). Boletim Epidemiológico AIDS DST. São Paulo: Secretaria de Estado da Saúde.

Silva, C. M., Jorge, A. S., Dalbosco, K., Peder, L. D. de, Horvath, J. D., \& Teixeira J. J. V, et al. (2017). Perfil epidemiológico dos pacientes com HIV em um centro de referência no sul do Brasil: características de dez anos. Rev Epidemiol e Control Infecção, 7(4), 227-233. https://doi.org/10.17058/reci.v7i4.9150 em 18 dez. 2021, de https://doi.org/10.1016/S2214-109X(15)00087-X

Zambenedetti, G., \& Silva, R. A. N. (2015). O paradoxo do território e os processos de estigmatização no acesso ao diagnóstico de HIV na atenção básica em saúde. Estud psicol. (Natal), 20(4), 229-40. https://doi.org/10.5935/1678-4669.20150024 